\title{
ESTIMATES FOR BELLMAN FUNCTIONS RELATED TO DYADIC-LIKE MAXIMAL OPERATORS ON WEIGHTED SPACES
}

\author{
ANTONIOS D. MELAS, ELEFTHERIOS N. NIKOLIDAKIS, AND DIMITRIOS CHELIOTIS
}

\begin{abstract}
We provide some new estimates for Bellman type functions for the dyadic maximal opeator $\mathbb{R}^{n}$ and of maximal operators on martingales related to weighted spaces. Using a type of symmetrization principle, introduced for the dyadic maximal operator in earlier works of the authors we introduce certain conditions on the weight that imply estimate for the maximal operator on the corresponding weighted space. Also using a well known estimate for the maximal operator by a double maximal operators on different measures related to the weight we give new estimates for the above Bellman type functions.
\end{abstract}

Acknowledgement 1. This research has been co-financed by the European Union and Greek national funds through the Operational Program "Education and Lifelong Learning" of the National Strategic Reference Framework (NSRF). ARISTEIA I, MAXBELLMAN 2760, research number 70/3/11913.

\section{INTRODUCTION}

The dyadic maximal operator on $\mathbb{R}^{n}$ is defined by

$$
M_{d} \phi(x)=\sup \left\{\frac{1}{|S|} \int_{S}|\phi(u)| d u: x \in S, S \subseteq \mathbb{R}^{n} \text { is a dyadic cube }\right\}
$$

for every $\phi \in L_{\text {loc }}^{1}\left(\mathbb{R}^{n}\right)$ where the dyadic cubes are the cubes formed by the grids $2^{-N} \mathbb{Z}^{n}$ for $N=0,1,2, \ldots$

As it is well known it satisfies the following weak $L^{p}$ inequality (for martingales known as Doob's inequality)

$$
\left\|M_{d} \phi\right\|_{p} \leq \frac{p}{p-1}\|\phi\|_{p}
$$

for every $p>1$ and every $\phi \in L^{p}\left(\mathbb{R}^{n}\right)$ which is best possible (see [1, 2] for the general martingales and 22] for dyadic ones).

An approach for studying more in depth the behavior of this maximal operator is the introduction of the so called Bellman functions (see [10]) related to them which reflect certain deeper properties of them by localizing. Such functions related to the $L^{p}$ inequality (1.2) have been precisely evaluated in [4]. Actually defining for any $p>1$

$$
\mathcal{B}_{p}(F, f)=\sup \left\{\frac{1}{|Q|} \int_{Q}\left(M_{d}^{\prime} \phi\right)^{p}: \operatorname{Av}_{Q}\left(\phi^{p}\right)=F, \operatorname{Av}_{Q}(\phi)=f\right\}
$$

Date: September 25, 2015.

2010 Mathematics Subject Classification. [2010] 42B25.

Key words and phrases. Bellman, dyadic, maximal, Lorentz. 
where $Q$ is a fixed dyadic cube, $R$ runs over all dyadic cubes containing $Q, \phi$ is nonnegative in $L^{p}(Q)$ and the variables $F, f$ satisfy $0 \leq f, f^{p} \leq F$ which is independent of the choice of $Q$ (so we may take $Q=[0,1]^{n}$ ) and where the localized maximal operator $M_{d}^{\prime} \phi$ is defined as in (1.1) with the dyadic cubes $S$ being assumed to be contained in the ambient dyadic cube $Q$. It has been shown in 4 that

$$
\mathcal{B}_{p}(F, f)=F \omega_{p}\left(\frac{f^{p}}{F}\right)^{p}
$$

where $\omega_{p}:[0,1] \rightarrow\left[1, \frac{p}{p-1}\right]$ is the inverse function of $H_{p}(z)=-(p-1) z^{p}+p z^{p-1}$. Actually (see [4]) the more general approach of defining Bellman functions with respect to the maximal operator on a nonatomic probability space $(X, \mu)$ equipped with a tree $\mathcal{T}$ (see Section 2) can be taken and the corresponding Bellman function is always the same. The fact that the range of $\omega_{p}$ is $\left[1, \frac{p}{p-1}\right]$ shows in a sense the extend that the constant in Doob's inequality can be approached only by functions whose integral is very small compared to its $p$-norm. For example for $p=2$ we get the following sharp improvement of Doob's inequality

$$
\left\|M_{\mathcal{T}} \phi\right\|_{2} \leq\|\phi\|_{2}+\left(\|\phi\|_{2}^{2}-\|\phi\|_{1}^{2}\right)^{1 / 2}<2\|\phi\|_{2}
$$

which aside from the $L^{2}$ norm of $\phi$ involves also in a sharp way the variance of $\phi$.

Here we will be concerned with the behavior of these maximal operators on weighted spaces. As it is well known for any positive locally integrable function $w$ on $Q$ the estimate

$$
\int_{Q}\left(M_{d}^{\prime} \phi\right)^{p} w \leq C \int_{Q} \phi^{p} w
$$

holds for all $\phi$ if and only if $w$ is a dyadic $A_{p}$ weight in the sense that

$$
\sup \left\{|I|^{-p}\left(\int_{I} w\right)\left(\int_{I} w^{-\frac{1}{p-1}}\right)^{p-1}: I \text { dyadic subcube of } Q\right\}=[w]_{p}<+\infty .
$$

Also it is known that the best possible $C$ is of the order of $[w]_{p}^{p /(p-1)}$, the exponent being best possible. Related to this one may define the following Bellman function given a weight $w$

$$
\mathcal{B}_{p, w}(F, f)=\sup \left\{\frac{1}{|Q|} \int_{Q}\left(M_{d}^{\prime} \phi\right)^{p} w: \operatorname{Av}_{Q}\left(\phi^{p} w\right)=F, \operatorname{Av}_{Q}(\phi)=f\right\}
$$

which is finite only if $w$ is in $A_{p}$ and seek estimates for this in order to improve the above estimate (1.6). One may add more variables to the above Bellman function as the integrals of $w$ and of $w^{-1 /(p-1)}$ over $Q$ but we will not treat those here. The estimates here will be proved in the general setting of tree like families on probability spaces and its related maximal operator, as will be described in the next section.

'We will derive two types of estimates related to the above problems. In the first we will use a related condition on some symmetrization of the weight to find the exact form of a related to weights Bellman function and this is done in section 2 . Then in section 3 we obtain certain new estimates for the above Bellman function related to $A_{p}$ with respect to a tree, and to the corresponding maximal operator, by using an estimate of the maximal operator via two applications of maximal operators on the same tree but with different measures, and this is described in section 3 . 
There are several other problems in Harmonic Analysis where Bellman functions naturally arise. Such problems (including the dyadic Carleson imbedding and weighted inequalities) are described in [12] (see also [10, 11]) and also connections to Stochastic Optimal Control are provided, from which it follows that the corresponding Bellman functions satisfy certain nonlinear second order PDE.

The exact computation of a Bellman function is a difficult task which is connected with the deeper structure of the corresponding Harmonic Analysis problem. Thus far several Bellman functions have been computed (see [1], 2], 4], 14], [15], 18], [19, 20]). L.Slavin and A.Stokolos [17] linked the Bellman function computation to solving certain PDE's of the Monge Ampere type, and in this way they obtained an alternative proof of the Bellman functions relate to the dyadic maximal operator in [4. Also in 20, using the Monge-Ampere equation approach a more general Bellman function than the one related to the dyadic Carleson imbedding Theorem has be precisely evaluated thus generalizing the corresponding result in [4].

\section{TREes, MAXIMAL OPERATORS AND SYMMETRIZATION}

As in [4] we let $(X, \mu)$ be a nonatomic probability space (i.e. $\mu(X)=1)$. Two measurable subsets $A, B$ of $X$ will be called almost disjoint if $\mu(A \cap B)=0$. Then we give the following.

Definition 1. A set $\mathcal{T}$ of measurable subsets of $X$ will be called a tree if the following conditions are satisfied:

(i) $X \in \mathcal{T}$ and for every $I \in \mathcal{T}$ we have $\mu(I)>0$.

(ii) For every $I \in \mathcal{T}$ there corresponds a finite subset $\mathcal{C}(I) \subseteq \mathcal{T}$ containing at least two elements such that:

(a) the elements of $\mathcal{C}(I)$ are pairwise almost disjoint subsets of $I$,

(b) $I=\bigcup \mathcal{C}(I)$.

(iii) $\mathcal{T}=\bigcup_{m \geq 0} \mathcal{T}_{(m)}$ where $\mathcal{T}_{(0)}=\{X\}$ and $\mathcal{T}_{(m+1)}=\bigcup_{I \in \mathcal{T}_{(m)}} \mathcal{C}(I)$.

(iv) We have $\lim _{m \rightarrow \infty} \sup _{I \in \mathcal{T}_{(m)}} \mu(I)=0$ and $\mathcal{T}$ differentiates $L^{1}$.

By removing the measure zero exceptional set $E(\mathcal{T})=\bigcup_{I \in \mathcal{T}} \bigcup_{\substack{J_{1}, J_{2} \in \mathcal{C}(I) \\ J_{1} \neq J_{2}}}\left(J_{1} \cap J_{2}\right)$ we may replace the almost disjointness above by disjointness.

Now given any tree $\mathcal{T}$ we define the maximal operator associated to it as follows

$$
M_{\mathcal{T}} \phi(x)=\sup \left\{\frac{1}{\mu(I)} \int_{I}|\phi| d \mu: x \in I \in \mathcal{T}\right\}
$$

for every $\phi \in L^{1}(X, \mu)$.

The above setting can be used not only for the dyadic maximal operator but also for the maximal operator on martingales, hence many of the results here can be viewed as generalizations and refinements of the classical Doob's inequality.

Also for any locally integrable positive function $w$ on $X$, which will be called weight, we denote $\sigma=w^{-\frac{1}{p-1}}$, and for any $I \in \mathcal{T}$ we write $w(I)=\int_{I} w d \mu, \sigma(I)=$ $\int_{I} \sigma d \mu$. Now we give the following.

Definition 2. A weight $w$ on $X$ will be called $A_{p}$ with respect to $\mathcal{T}$ if the following expression

$$
[w]_{\mathcal{T}, p}=[w]_{p}=\sup _{I \in \mathcal{T}} \frac{w(I) \sigma(I)^{p-1}}{\mu(I)^{p}}
$$


is finite.

A way to study estimates for the above maximal operator is through the symmetrization of $\phi$ as has been introduced in [5] and [13] and used in [8] to evaluate Bellman functions related to Lorentz norms. In order to apply this in the context of weights we introduce the following condition on a weight $w$ on $X$.

Definition 3. A weight $w$ on $X$ will be called $A_{p}^{*}$ if for some equimeasurable rearrrangement $w^{* *}$ of $w$ on $(0,1)$ (not necessarily decreasing) there exist two constants $c, a>0$ such that for every $t$ in $(0,1]$ the following estimate holds

$$
\int_{t}^{1} \frac{w^{* *}(s)}{s^{p}} d s+c \leq a \frac{w^{* *}(t)}{t^{p-1}}
$$

and also

$$
\lim _{t \rightarrow 0^{+}} t^{p} \int_{t}^{1} \frac{w^{* *}(s)}{s^{p}} d s=0
$$

Note that by writing $r(t)=\frac{w^{* *}(t)}{t^{p-1}}$ the first condition implies that $r(t)>\frac{c}{a}>0$ for all $t$ hence $\lim _{t \rightarrow 0^{+}} \int_{t}^{1} \frac{w^{* *}(s)}{s^{p}} d s=+\infty$ and so $\lim _{t \rightarrow 0^{+}} r(t)=0$. Hence we conclude that there is a best possible pair $(a, c)$ for each such weight, namely $a=$ $\sup _{t} r(t)^{-1} \int_{t}^{1} \frac{r(t)}{t} d t$ and $c=\sup _{t}\left(\operatorname{ar}(t)-\int_{t}^{1} \frac{r(t)}{t} d t\right)$. We will refer to this pair as the constants of the corresponding $A_{p}^{*}$ weight $w$.

Example. Suppose that $w^{* *}(t)=k t^{b}$ with $k, b \in \mathbf{R}$. Then the above conditions hold if and only if $-1<b<p-1$ which is exactly the range making $w^{* *}$ an $A_{p}$ weight on $(0,1)$. Moreover the corresponding constants $c, a$ can be easily seen to be $a=\frac{1}{p-1-b}, c=\frac{k}{p-1-b}$.

Now we take into consideration the following theorem proved in 13 and [5].

Theorem 1. Let $G:[0,+\infty) \rightarrow[0,+\infty)$ be non-decrasing, $h:(0,1] \rightarrow \mathbb{R}^{+}$be any locally integrable function. Then for any nonatomic probability space $(X, \mu)$, equipped with any tree-like family $\mathcal{T}$, for any non-increasing right continuous integrable function $g:(0,1] \rightarrow \mathbb{R}^{+}$and any $k \in(0,1]$, the following equality holds (where by $\psi^{*}$ we denote the decreasing equimeasurable rearrangement of $\psi$ ):en

$$
\begin{gathered}
\sup \left\{\int_{0}^{k} G\left[\left(M_{\mathcal{T}} \phi\right)^{*}(t)\right] h(t) d t: \phi \text { measurable on } X \text { with } \phi^{*}=g\right\}= \\
=\int_{0}^{k} G\left(\frac{1}{t} \int_{0}^{t} g(u) d u\right) h(t) d t .
\end{gathered}
$$

After this given an $A_{p}^{*}$ weight $w$, we define the following variant of the Bellman function (1.7).

$$
\mathcal{B}_{p, w}^{*}(F, f)=\sup \left\{\int_{0}^{1}\left(\left(M_{\mathcal{T}} \phi\right)^{*}\right)^{p} w^{* *}: \int_{0}^{1}\left(\phi^{*}\right)^{p} w^{* *}=F, \int_{X} \phi=f\right\}
$$

where here by $\phi^{*},\left(M_{\mathcal{T}} \phi\right)^{*}$ we denote the equimeasurable decreasing rearrangement of $\phi$ and $M_{\mathcal{T}} \phi$ whereas by $w^{* *}$ we denote the equimeasurable rearrangement of $w$ that appears in the above definition. Note that in case $w^{* *}$ is decreasing $\int_{X}\left(\left(M_{\mathcal{T}} \phi\right)^{*}\right)^{p} w^{* *}$ is greater than or equal to $\int_{X}\left(M_{\mathcal{T}} \phi\right)^{p} w$ and $\int_{0}^{1}\left(\phi^{*}\right)^{p} w^{* *}$ is greater 
than or equal to $\int_{X} \phi^{p} w$ and when $w^{* *}$ is increasing then the opposite relations hold. Then we can prove the following.

Theorem 2. For the above function we have

$$
\mathcal{B}_{p, w}^{*}(F, f)=(p-1)^{p} a^{p} F \omega_{p}\left(\frac{c f^{p}}{(p-1)^{p-1} a^{p} F}\right)^{p} .
$$

where $c$, a are the constants of the $A_{p}^{*}$ weight $w$, the domain of this function being all $(F, f)$ such that $c f^{p} \leq(p-1)^{p-1} a^{p} F$.

Proof. In view of the above mentioned result it suffices to consider the expression $\Delta_{w}(g)=\int_{0}^{1}\left(t^{-1} \int_{0}^{t} g(u) d u\right)^{p} w^{* *}(t) d t$ when $g$ runs over all nonnegative decreasing right continuous functions on $(0,1]$ satisfying $\int_{0}^{1} g(t) d t=f$ and $\int_{0}^{1} g(t)^{p} w^{* *}(t) d t=$ $F$. We next define the following function on $(0,1)$

$$
u(t)=\int_{t}^{1} \frac{w^{* *}(s)}{s^{p}} d s+c
$$

so that $u^{\prime}(t)=t^{-p} w^{* *}(t)$. Considering first any bounded such function $g$ we compute by integration by parts

$$
\begin{aligned}
& \int_{0}^{1} u(t)\left(\int_{0}^{t} g(u) d u\right)^{p-1} g(t) d t=\frac{1}{p} \int_{0}^{1} u(t)\left[\left(\int_{0}^{t} g(u) d u\right)^{p}\right]^{\prime} d t= \\
= & \frac{1}{p}\left(\int_{0}^{1} g(u) d u\right)^{p} u(1)+\frac{1}{p} \int_{0}^{1}\left(t^{-1} \int_{0}^{t} g(u) d u\right)^{p} w^{* *}(t)=c \frac{f^{q}}{p}+\frac{1}{p} \Delta(g)
\end{aligned}
$$

the integration by parts term $\lim _{t \rightarrow 0+} u(t)\left(\int_{0}^{t} g(u) d u\right)^{p}$ being zero because of condition (2.3) since $g$ is assumed bounded. Now using Young's inequality $x y \leq \frac{x p}{p}+\frac{y^{p^{\prime}}}{p^{\prime}}$ (where $\left.p^{\prime}=p /(p-1)\right)$ in the first integral as follows, $(\lambda>0$ to be determined later) combined with the condition $\frac{u(t) t^{p-1}}{w^{* *}(t)} \leq a$ from the above definition we get

$$
\begin{gathered}
\int_{0}^{1} u(t)\left(\int_{0}^{t} g(u) d u\right)^{p-1} g(t) d t= \\
=\int_{0}^{1}\left(\lambda g(t) w^{* *}(t)^{1 / p}\right)\left(\frac{w^{* *}(t)^{1 / p}}{\lambda^{1 /(p-1)} t} \int_{0}^{t} g(u) d u\right)^{p-1} \frac{u(t) t^{p-1}}{w^{* *}(t)} d t \leq \\
\leq \frac{a}{p} \int_{0}^{1} \lambda^{p} g(t)^{p} w^{* *}(t) d t+\frac{a}{p^{\prime}} \int_{0}^{1} \lambda^{-p^{\prime}}\left(\frac{1}{t} \int_{0}^{t} g(u) d u\right)^{p} w^{* *}(t) d t= \\
=\frac{a \lambda^{p}}{p} \int_{0}^{1} g(t)^{p} w^{* *}(t) d t+a \frac{\lambda^{-p^{\prime}}}{p^{\prime}} \int_{0}^{1}\left(\frac{1}{t} \int_{0}^{t} g(u) d u\right)^{p} w^{* *}(t) d t=a \frac{\lambda^{p}}{p} F+a \frac{\lambda^{-p^{\prime}}}{p^{\prime}} \Delta_{w}(g) .
\end{gathered}
$$

Therefore we have by writing $\lambda^{p^{\prime}}=(p-1) a(\beta+1), \beta>0$ and using the above inequalities we get that

$$
\Delta_{w}(g) \leq\left(1+\frac{1}{\beta}\right) \frac{(\beta+1)^{p-1}(p-1)^{p} a^{p} F-(p-1) c f^{p}}{(p-1)}
$$

Next, given an arbitrary $g$, the above estimate can be used for the truncations $g_{M}=\min (g, M)$ and $F, f$ replaced by the corresponding quantities for $g_{M}$ and then take $M \rightarrow+\infty$ and use monotone convergence to infer that (2.5) holds for the general nonnegative decreasing right continuous function on $(0,1]$ satisfying $\int_{0}^{1} g(t) d t=f$ and $\int_{0}^{1} g(t)^{p} w^{* *}(t) d t=F$. Moreover since $\Delta_{w}(g)>0$ the inequality 
(2.5) implies that $(\beta+1)^{p-1}(p-1)^{p} a^{p} F-(p-1) c f^{p}>0$ for every $\beta>0$ and so letting $\beta \rightarrow 0^{+}$we conclude that $(F, f)$ must satisfy the inequality $c f^{p} \leq(p-1)^{p-1} a^{p} F$ given in the statement of the Theorem.

Writing $A=(p-1)^{p} a^{p} F$ and $B=(p-1) c f^{p}$ it is easy to compute (see for example 4 pg. 326) that the minimum possible value of the right hand side of (2.5) is equal to $A \omega_{p}\left(\frac{B}{A}\right)^{p}$. This proves the inequality

$$
\mathcal{B}_{p, w}^{*}(F, f) \leq(p-1)^{p} a^{p} F \omega_{p}\left(\frac{c f^{p}}{(p-1)^{p-1} a^{p} F}\right)^{p} .
$$

Now we consider the continuous positive decreasing function

$$
g_{\alpha}(t)=f(1-\alpha) t^{-\alpha}
$$

where $0 \leq \alpha<1$, and any $A_{p}^{*}$ weight $w$ that is equimeasurable to

$$
w^{* *}(t)=k t^{b}, k>0,-1<b<p-1
$$

Clearly $\int_{0}^{1} g_{\alpha}(t) d t=f$ and $\int_{0}^{1} g_{\alpha}(t)^{p} w^{* *}(t) d t=\frac{k f^{p}(1-\alpha)^{p}}{1+b-\alpha p}$ assuming that $\alpha<\frac{1+b}{p}$. Next note that $\frac{1}{t} \int_{0}^{t} g_{\alpha}(u) d u=\frac{g_{\alpha}(t)}{1-\alpha}$ for all $t \in(0,1]$ and so we have $\Delta_{w}\left(g_{\alpha}\right)=$ $\left(\frac{1}{1-\alpha}\right)^{p} \int_{0}^{1} g_{\alpha}(t)^{p} w^{* *}(t) d t$. The condition $\int_{0}^{1} g_{\alpha}(t)^{p} w^{* *}(t) d t=F$ is then equivalent to the following equation in $\alpha$

$$
\frac{(1-\alpha)^{p}}{1+b-\alpha p}=\frac{F}{k f^{q}}
$$

To study this equation we write

$$
z=\frac{p-1-b}{p-1} \frac{1}{1-\alpha}
$$

and note that (2.9) is then equivalent to

$$
-(p-1) z^{p}+p z^{p-1}=\frac{k f^{p}}{\left(\frac{p-1}{p-1-b}\right)^{p-1} F}
$$

thus

$$
z=\omega_{p}\left(\frac{k f^{p}}{\left(\frac{p-1}{p-1-b}\right)^{p-1} F}\right)
$$

and so using (2.10)

$$
\Delta_{w}\left(g_{\alpha}\right)=\left(\frac{p-1}{p-1-b}\right)^{p} F \omega_{p}\left(\frac{k f^{p}}{\left(\frac{p-1}{p-1-b}\right)^{p-1} F}\right) .
$$

But now note that the constants $c, a$ of the weight $w$ are $a=\frac{1}{p-1-b}, c=\frac{k}{p-1-b}$ and so

$$
\Delta_{w}\left(g_{\alpha}\right)=(p-1)^{p} a^{p} F \omega_{p}\left(\frac{c f^{p}}{(p-1)^{p-1} a^{p} F}\right)^{p}
$$

and moreover by varying $k, b$ with $-1<b<p-1$ we can achieve all possible pairs of constants $c, a$. This completes the proof. 


\section{Estimation Via Double MAXimal operators}

Here we will use an inequality introduced by A. Lerner, see [3], for the nondyadic case. We fix $p>1$, let $w$ be an $A_{p}$ weight with respect to the tree $\mathcal{T}$ and we denote for any $I$ in $\mathcal{T}, w(I)=\int_{I} w d \mu, \sigma=w^{-\frac{1}{p-1}}, \sigma(I)=\int_{I} \sigma d \mu$. Also by $M_{\mathcal{T}, w}$ we denote the maximal operator with respect to the tree $\mathcal{T}$ but when $X$ is equipped by the measure $w \mu$ instead of $\mu$, and similarly for $M_{\mathcal{T}, \sigma}$. Then the following holds.

Proposition 1. Let $w$ be an $A_{p}$ weight with respect to the tree $\mathcal{T}$ and $\mathcal{T}$-constant $[w]_{p}=\sup _{I \in \mathcal{T}} \frac{w(I) \sigma(I)^{p-1}}{\mu(I)^{p}}$ Then for any $\phi$ we have the following pointwise estimate

$$
\left(M_{\mathcal{T}} \phi\right)^{p-1} \leq[w]_{p} M_{\mathcal{T}, w}\left[\left(M_{\mathcal{T}, \sigma}\left(\phi \sigma^{-1}\right)\right)^{p-1} w^{-1}\right]
$$

Proof. The proof follows from the following inequalities valid for any $I \in \mathcal{T}$.

$$
\begin{aligned}
\left(\frac{1}{\mu(I)} \int_{I} \phi d \mu\right)^{p-1} & =\frac{w(I) \sigma(I)^{p-1}}{\mu(I)^{p}}\left(\frac{\mu(I)}{w(I)}\left(\frac{1}{\sigma(I)} \int_{I} \phi \sigma^{-1} \sigma d \mu\right)^{p-1}\right) \leq \\
& \leq[w]_{p} \frac{1}{w(I)} \int_{I} M_{\mathcal{T}, \sigma}\left(\phi \sigma^{-1}\right)^{p-1} w^{-1} w d \mu
\end{aligned}
$$

since $M_{\mathcal{T}, \sigma}\left(\phi \sigma^{-1}\right)(x) \geq \frac{1}{\sigma(I)} \int_{I} \phi \sigma^{-1} \sigma d \mu$ for every $x$ in $I$.

As a first application of this fixing a tree $\mathcal{T}$ on a probability space $(X, \mu)$ and given an $A_{p}$ weight $w$ in the sense of Definition 2, we define the following generalization of the Bellman function (1.7), where $p>1$

$$
\mathcal{B}_{p, w}^{\mathcal{T}}(F, f)=\sup \left\{\int_{X}\left(M_{\mathcal{T}} \phi\right)^{p} w d \mu: \int_{X} \phi^{p} w d \mu=F, \int_{X} \phi d \mu=f\right\}
$$

and we have the following estimates

Theorem 3. For any tree $\mathcal{T}$ on a probability space $(X, \mu)$ and any $A_{p}$ weight $w$ and any $\phi$ with $\int_{X} \phi^{p} w d \mu=F, \int_{X} \phi d \mu=f$ we have

$$
\begin{gathered}
\int_{X}\left(M_{\mathcal{T}} \phi\right)^{p} w d \mu \leq \\
\leq[w]_{p}^{1 /(p-1)} F \omega_{p}\left(\frac{f^{p}}{\sigma(X)^{p-1} F}\right)^{p} \omega_{p^{\prime}}\left(\frac{\left(\int_{X} \phi^{p-1} w d \mu\right)^{p^{\prime}}}{w(X)^{p^{\prime}-1} F \omega_{p}\left(\frac{f^{p}}{\sigma(X)^{p-1} F}\right)^{p}}\right)^{p^{\prime}}
\end{gathered}
$$

In particular

$$
\mathcal{B}_{p, w}^{\mathcal{T}}(F, f) \leq p^{p^{\prime}}[w]_{p}^{1 /(p-1)} F \omega_{p}\left(\frac{f^{p}}{\sigma(X)^{p-1} F}\right)^{p}
$$

Proof. By applying estimate (1.12) after Theorem 1 in 4 for the exponent $p^{\prime}=\frac{p}{p-1}$ to the function $\rho=\left(M_{\mathcal{T}, \sigma}\left(\phi \sigma^{-1}\right)\right)^{p-1} w^{-1}$ and with respect to the tree $\mathcal{T}$ but on 
the probability space $\left(X, \frac{1}{w(X)} w d \mu\right)$ (where as usual $\left.w(X)=\int_{X} w d \mu\right)$ we get

$$
\begin{gathered}
\frac{1}{[w]_{p}^{1 /(p-1)}} \int_{X}\left(M_{\mathcal{T}} \phi\right)^{p} w d \mu \leq w(X) \int_{X}\left(M_{\mathcal{T}, w} \rho\right)^{p^{\prime}} w \frac{d \mu}{w(X)} \leq \\
\leq w(X) \int_{X} \rho^{p^{\prime}} w \frac{d \mu}{w(X)} \cdot \omega_{p^{\prime}}\left(\frac{\left(\int_{X} \rho w \frac{d \mu}{w(X)}\right)^{p^{\prime}}}{\int_{X} \rho^{p^{\prime}} w \frac{d \mu}{w(X)}}\right)^{p^{\prime}} .
\end{gathered}
$$

Note that (as proved in [4]) the function $x \omega_{p^{\prime}}\left(\frac{y^{p^{\prime}}}{x}\right)^{p^{\prime}}$ is increasing in $x$ and decreasing in $y$. Now we have

$$
\int_{X} \rho w \frac{d \mu}{w(X)}=\int_{X}\left(M_{\mathcal{T}, \sigma}\left(\phi \sigma^{-1}\right)\right)^{p-1} \frac{d \mu}{w(X)} \geq \int_{X}\left(\phi \sigma^{-1}\right)^{p-1} \frac{d \mu}{w(X)}=\int_{X} \phi^{p-1} w \frac{d \mu}{w(X)}
$$

and using estimate (1.12) after Theorem 1 in [4] for the exponent $p$ to the function $\rho=\phi \sigma^{-1}$ and with respect to the tree $\mathcal{T}$ but on the probability space $\left(X, \frac{1}{\sigma(X)} \sigma d \mu\right)$ we get (since $\sigma^{-(p-1)}=w$ )

$$
\begin{gathered}
\int_{X} \rho^{p^{\prime}} w d \mu=\int_{X}\left(M_{\mathcal{T}, \sigma}\left(\phi \sigma^{-1}\right)\right)^{p} w^{-p^{\prime}} \cdot w d \mu=\int_{X}\left(M_{\mathcal{T}, \sigma}\left(\phi \sigma^{-1}\right)\right)^{p} \sigma d \mu \leq \\
\sigma(X) \int_{X}\left(\phi \sigma^{-1}\right)^{p} \sigma \frac{d \mu}{\sigma(X)} \cdot \omega_{p}\left(\frac{\left(\int_{X} \phi \sigma^{-1} \sigma \frac{d \mu}{\sigma(X)}\right)^{p}}{\int_{X}\left(\phi \sigma^{-1}\right)^{p} \sigma \frac{d \mu}{\sigma(X)}}\right)^{p}= \\
=\int_{X} \phi^{p} w d \mu \cdot \omega_{p}\left(\frac{\left(\int_{X} \phi d \mu\right)^{p}}{\sigma(X)^{p-1} \int_{X} \phi^{p} w d \mu}\right)^{p}=F \omega_{p}\left(\frac{f^{p}}{\sigma(X)^{p-1} F}\right)^{p} .
\end{gathered}
$$

Now combining the above estimates we get

$$
\begin{gathered}
\frac{1}{[w]_{p}^{1 /(p-1)}} \int_{X}\left(M_{\mathcal{T}} \phi\right)^{p} w d \mu \leq \\
\leq F \omega_{p}\left(\frac{f^{p}}{\sigma(X)^{p-1} F}\right)^{p} \omega_{p^{\prime}}\left(\frac{\left(\int_{X} \phi^{p-1} w d \mu\right)^{p^{\prime}}}{w(X)^{p^{\prime}-1} F \omega_{p}\left(\frac{f^{p}}{\sigma(X)^{p-1} F}\right)^{p}}\right)^{p^{\prime}}
\end{gathered}
$$

which proves (3.2). Since $\omega_{p^{\prime}}(x) \leq \frac{p^{\prime}}{p^{\prime}-1}=p$ the estimate (3.3) follows also.

To get lower bounds for the Bellman function we invoke the following construction.

Fixing $\alpha$ with $0<\alpha<1$ and using Lemma 1 in 4 , we fix now a tree $\mathcal{T}$, for example the dyadic subintervals of $[0,1]$, and choose for every $I \in \mathcal{T}$ a family $\mathcal{F}(I) \subseteq \mathcal{T}$ of pairwise almost disjoint subsets of $I$ such that

$$
\sum_{J \in \mathcal{F}(I)} \mu(J)=(1-\alpha) \mu(I) .
$$

Then we define $\mathcal{S}=\mathcal{S}_{\alpha}$ to be the smallest subset of $\mathcal{T}$ such that $X \in \mathcal{S}$ and for every $I \in \mathcal{S}, \mathcal{F}(I) \subseteq \mathcal{S}$. Next for every $I \in \mathcal{S}$ we define the set

$$
A_{I}=I \backslash \bigcup_{J \in \mathcal{F}(I)} J
$$


and note that $\mu\left(A_{I}\right)=\alpha \mu(I)$ and $I=\bigcup_{\substack{J \in \mathcal{S} \\ J \subseteq I}} A_{J}$ for every $I \in \mathcal{S}$. Also since $\mathcal{S}=$ $\bigcup_{m \geq 0} \mathcal{S}_{(m)}$ where $\mathcal{S}_{(0)}=\{X\}$ and $\mathcal{S}_{(m+1)}=\bigcup_{I \in \mathcal{S}_{(m)}} \mathcal{F}(I)$, we can define $\operatorname{rank}(I)=$ $r(I)$ for $I \in \mathcal{S}$ to be the unique integer $m$ such that $I \in \mathcal{S}_{(m)}$ and remark that $\sum_{\substack{\mathcal{S} \ni J \subseteq I \\ \hdashline}} \mu(J)=(1-\alpha)^{m} \mu(I)$ for every $I \in \mathcal{S}$. $r(J)=r(I)+m$

Next for any $\lambda, \gamma>0$ we define the function

$$
\psi=\sum_{I \in \mathcal{S}} \lambda \gamma^{r(I)} \chi_{A_{I}}
$$

and we have for any $I \in \mathcal{S}$ the following

$$
\frac{1}{\mu(I)} \int_{I} \psi d \mu=\frac{\lambda \alpha}{1-\gamma(1-\alpha)} \gamma^{r(I)} .
$$

Hence taking

$$
\phi_{\alpha}=\sum_{I \in \mathcal{S}} \lambda_{1} \gamma_{1}^{r(I)} \chi_{A_{I}}, \quad w_{\alpha}=\sum_{I \in \mathcal{S}} \lambda_{2} \gamma_{2}^{r(I)} \chi_{A_{I}}
$$

we have for any $I \in \mathcal{S}$

$$
\frac{w_{\alpha}(I)\left[w_{\alpha}^{-\frac{1}{p-1}}(I)\right]^{p-1}}{\mu(I)^{p}}=\frac{\alpha^{p}}{\left[1-\gamma_{2}(1-\alpha)\right]\left[1-\gamma_{2}^{-\frac{1}{p-1}}(1-\alpha)\right]}
$$

thus $w_{\alpha}$ is an $A_{p}$ weight but with respect to the tree $\mathcal{S}_{\alpha}$ on $(X, \mu)$ and with $\left[w_{\alpha}\right]_{p}$ equal to the right hand side of the above relation. Moreover

$$
M_{\mathcal{S}} \phi_{\alpha} \geq \sum_{I \in \mathcal{S}} \frac{1}{\mu(I)} \int_{I} \phi_{a} d \mu \chi_{A_{I}}=\frac{\alpha}{1-\gamma(1-\alpha)} \phi_{\alpha} .
$$

However the values of such functions on each $A_{I}$ where $r(I)=m$ is of the form

$$
\gamma_{m}=\frac{\lambda}{\alpha(1-\alpha)^{m}} \int_{(1-\alpha)^{m+1}}^{(1-\alpha)^{m}} u^{s} d u
$$

for some real numbers $\lambda, s>0$ and as it is proved in Lemma 3 of 8 these behave like functions of the form $\lambda t^{s}$ on $(0,1]$ as we approach the limit $\alpha \rightarrow 0^{+}$. Hence by taking a sequence $\alpha_{m} \rightarrow 0$ considering the trees $\mathcal{T}_{m}=\mathcal{S}_{\alpha_{m}}$ on $(X, \mu)$ and using the construction for the lower bound in the proof of Theorem 2, choosing the constants $k, b(-1<b<p-1)$ appropriately in (2.8) according to the conditions $a=\frac{1}{p-1-b}, c=k a, \frac{k}{b+1}=z, \frac{1}{b+1}\left(\frac{p-1}{p-1-b}\right)^{p-1}=h$ from the restrictions below which give $\frac{p-1-b}{p-1}=\omega_{p}\left(\frac{1}{h}\right)$ we conclude the following.

Proposition 2. Given appropriate $F, f, h, z$ there exists a sequence of trees $\mathcal{T}_{m}$ on $(X, \mu)$ and two sequences $\left(\phi_{m}\right)$ and $\left(w_{m}\right)$ of positive measurable functions on $(X, \mu)$ such that $\int_{X} \phi_{m} d \mu \rightarrow f, \int_{X} \phi_{m}^{p} w_{m} d \mu \rightarrow F$, each $w_{m}$ is an $A_{p}$ weight with respect to the tree $\mathcal{T}_{m}$ with $\left[w_{m}\right]_{p} \rightarrow h$ and $\int_{X} w_{m} d \mu \rightarrow z$ such that

$$
\lim _{m \rightarrow \infty} \int_{X}\left(M_{\mathcal{T}_{m}} \phi_{m}\right)^{p} w_{m} d \mu \geq F \omega_{p}\left(\frac{z f^{p}}{h F}\right)^{p} \omega_{p}\left(\frac{1}{h}\right)^{-p} .
$$

The above proposition implies a lower bound on the class of functions $\mathcal{B}_{p, w}^{\mathcal{T}}(F, f)$ when viewed over all trees $\mathcal{T}$ and corresponding $A_{p}$ weights $w$. 


\section{REFERENCES}

[1] D. L. Burkholder, Martingales and Fourier analysis in Banach spaces, C.I.M.E. Lectures (Varenna (Como), Italy, 1985), Lecture Notes in Mathematics 1206 (1986), 61-108.

[2] D. L. Burkholder, Boundary value problems and sharp inequalities for martingale transforms, Ann. of Prob. 12 (1984), 647-702.

[3] A. K. Lerner, An elementary approach to several results on the Hardy-Littlewood maximal operator, Proc. Amer. Math. Soc. 136 (2008), no.8, 2829-2833.

[4] A. D. Melas, The Bellman functions of dyadic-like maximal operators and related inequalities, Adv. in Math. 192 (2005), 310-340.

[5] A. D. Melas, Sharp general local estimates for dyadic -like maximal operators and related Bellman functions, Adv. in Math. 220 (2009), 1631-1654.

[6] A. D. Melas, Dyadic-like maximal operators on $L \log L$ functions, Journal of Funct. Anal. 257 no. 6 (2009), 367-426.

[7] A. D. Melas, E. Nikolidakis, A sharp integral rearrangement inequality for the dyadic maximal operator and applications, submitted.

[8] A. D. Melas, E. Nikolidakis, Sharp Lorentz estimates for dyadic-like maximal operators and related Bellman functions, submitted.

[9] A. D. Melas, E. Nikolidakis, Th. Stavropoulos, Sharp local lower $L^{p}$-bounds for dyadic-like maximal operators, Proc.. Amer. Math. Soc. 141 (2013), 3171-3181.

[10] F. Nazarov, S. Treil, The hunt for a Bellman function: applications to estimates for singular integral operators and to other classical problems of harmonic analysis, Algebra $i$ Analyz 8 no. 5 (1996), 32-162.

[11] F. Nazarov, S. Treil, A. Volberg, The Bellman functions and two-weight inequalities for Haar multipliers. Journ. Amer. Math. Soc. 12 no. 4 (1999), 909-928.

[12] F. Nazarov, S. Treil, A. Volberg, Bellman function in Stochastic Optimal Control and Harmonic Analysis (how our Bellman function got its name), Oper. Theory: Advances and Appl. 129 (2001), 393-424, Birkhäuser Verlag.

[13] E. Nikolidakis, The geometry of the maximal operator, Rev. Math.Iber., to appear.

[14] L. Slavin, V. Vasyunin, Sharp results in the integral-form John-Nirenberg inequality, Trans. Amer. Math. Soc. 363, no 8, (2011), 4135-4169.

[15] L. Slavin, A. Volberg. The explicit BF for a dyadic Chang-Wilson-Wolff theorem. The sfunction and the exponential integral. Contmp. Math., 444 (2007), 215-228.

[16] E. M. Stein. Harmonic Analysis, Princeton Mathematical Series 43, (1993), Princeton University Press.

[17] L. Slavin, A. Stokolos, V. Vasyunin. Monge-Ampère equations and Bellman functions: The dyadic maximal operator. C. R. Acad. Paris Sér. I Math., 346, no 9-10, (2008), 585-588.

[18] V. Vasyunin. The sharp constant in the reverse Holder inequality for Muckenhoupt weights. Algebra i Analiz, 15 (2003), no. 1, 73-117

[19] V. Vasyunin, A. Volberg. The Bellman functions for a certain two weight inequality: the case study. Algebra i Analiz, 18 (2006), No. 2

[20] V. Vasyunin, A. Volberg. Monge-Ampere equation and Bellman optimization of Carleson embedding Theorem, Amer. Math. Soc. Translations Series 2, 226, (2009).

[21] A. Volberg, Bellman approach to some problems in Harmonic Analysis, Seminaire des Equations aux derivées partielles, Ecole Polytéchnique, 2002, eposé. XX, 1-14.

[22] G. Wang, Sharp maximal inequalities for conditionally symmetric martingales and Brownian motion, Proc. Amer. Math. Soc. 112 (1991), 579-586.

Department of Mathematics, University of Athens, Panepistimiopolis 15784, Athens, Greece

E-mail address: amelas@math.uoa.gr, lefteris@math.uoc.gr, dcheliotis@math.uoa.gr 